\title{
Diseño de ecocubiertas en material plástico reciclado reforzado con fibra de fique (Furcraea andina) para mejorar las condiciones de habitabilidad de las viviendas del sector rural colombiano
}

María Paula Aranzales-Sánchez ${ }^{1}$

Universidad Piloto de Colombia - Seccional Alto Magdalena mariaaranzales@gmail.com

DOI: https://doi.org/10.21158/23823399.v8.n0.2020.2616

Fecha de recepción: 12 de marzo de 2020 Fecha de aprobación: 08 de junio de 2020

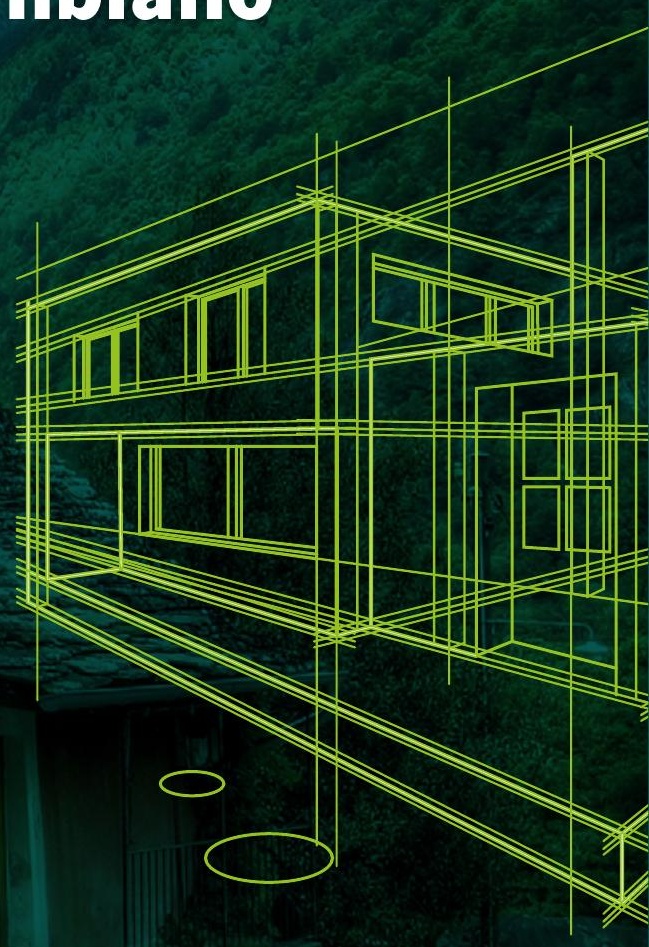

Cómo citar este artículo: Aranzales-Sánchez, M. P. (2020). Diseño de ecocubiertas en material plástico reciclado reforzado con fibra de fique (Furcraea andina) para mejorar las condiciones de habitabilidad de las viviendas del sector rural colombiano. Revista Ontare, 8, 11-30.

DOI: https://doi.org/10.21158/23823399.v8.n0.2020.2616

${ }^{1}$ Estudiante del programa de Ingeniería Civil de la Universidad Piloto de Colombia - Seccional Alto Magdalena. Joven investigadora,
perteneciente al semillero de investigación Sentram de transporte masivo, energías renovables y materiales bioconstructivos. ORCID: https://orcid.org/0000-0002-5416-927X 



\section{RESUMEN}

El presente trabajo de investigación tiene su origen en dos problemáticas fundamentales en la actualidad: la contaminación y los impactos ambientales generados por la acumulación masiva de residuos plásticos y materiales de construcción; y el déficit de viviendas y los problemas de calidad de materiales y estructuración en estas, principalmente en las poblaciones rurales de Colombia víctimas de desplazamiento, violencia, fenómenos naturales y falta de atención e inversión por parte de los entes gubernamentales. Entonces, ante la necesidad de ahondar en la tecnología de las materias primas para generar nuevos materiales, el objetivo de este texto fue estudiar el uso de termoplásticos posconsumo fabricados en polietileno de alta densidad, cloruro de polivinilo y polietileno tereftalato, reforzados con fibra de fique, en la fabricación de elementos de construcción, con el propósito de mejorar las condiciones estructurales y de habitabilidad en las viviendas rurales. Se implementó una metodología de carácter documental y científico con método experimental y enfoque mixto, en la que luego de un proceso de investigación sobre las propiedades físicas y mecánicas de los materiales se desarrollaron seis prototipos con distintas proporciones, resultantes de la fusión de los plásticos reciclados y la fibra de fique, determinando el comportamiento mecánico de los compuestos a partir de ensayos de resistencia a la tracción, la compresión, la flexión, el calor y la estabilidad. Así, se determinó que la fibra no aumenta la resistencia, pero sí la capacidad de deformarse sin incurrir en la rotura, lo que le brinda amplias propiedades de ductilidad.

Palabras clave: uso de residuos plásticos; termoplásticos; posconsumo; polietileno de alta densidad; cloruro de polivinilo; polietileno tereftalato; materiales de construcción; vivienda rural. 


\section{Design of ecocovers in recycled plastic material reinforced with fique fiber (Furcraea andina) to improve housing conditions in the rural areas in Colombia}

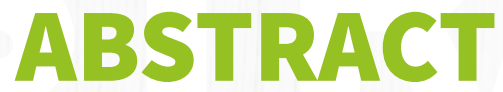

This research work derives from two fundamental problems that are currently taking place: the pollution and environmental impacts generated by the massive accumulation of plastic waste and construction materials, and the housing deficit, the structural situation and the problems related to the quality of the materials that are being used to build them; mainly in the rural populations of Colombia that are subjected to displacement, violence, natural phenomena, and lack of attention and investment by government bodies. Therefore, in view of the need to go deeper into the technology of raw materials to generate new materials, the objective of this text was to study the use of post-consumer thermoplastics made of high-density polyethylene, polyvinyl chloride, and polyethylene terephthalate, reinforced with fique fiber, in the manufacture of construction elements, with the purpose of improving the structural and habitability conditions in rural housing. We implemented a documentary and scientific methodology with an experimental method and mixed approach, and after a research process on the physical and mechanical properties of the materials, six prototypes with different proportions were developed, resulting from the fusion of recycled plastics and fique fiber, determining the mechanical behavior of the compounds from material resistance testing to traction, compression, flexion, heat, and stability. Thus, it was determined that the fiber does not increase resistance, but it does increase the capacity of deformation without incurring in the breakage, which gives it wide properties of ductility.

Keywords: use of plastic waste; thermoplastics; post-consumption; high density polyethylene; polyvinyl chloride; polyethylene terephthalate; building materials; rural housing. 


\section{Desenho de ecocoberturas em material plástico reciclado reforçado com fibra de fique (Furcraea andina) para melhorar as condições de habitabilidade das moradias do setor rural colombiano}

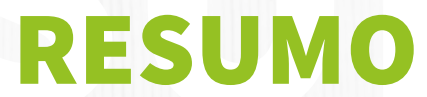

0 presente trabalho de pesquisa tem sua origem em duas problemáticas fundamentais da atualidade: a contaminação e os impactos ambientais gerados pela acumulação em massa de resíduos plásticos e materiais de construção; e o déficit de moradias e os problemas de qualidade de materiais e estrutura destas, principalmente em populações rurais da Colômbia vítimas de deslocação, violência, fenômenos naturais e falta de atenção e investimento por parte dos entes governamentais. Assim, ante a necessidade de aprofundar a tecnologia das matérias prima para gerar novos materiais, o objetivo deste texto foi estudar o uso de termoplásticos pós-consumo fabricados em polietileno de alta densidade, cloruro de polivinilo e polietileno tereftalato, reforçados com fibra de fique, na fabricação de elementos de construção, com o propósito de melhorar as condições estruturais e de habitabilidade nas moradias rurais. Implementou-se uma metodologia de caráter documental e científico com método experimental e enfoque misto, na qual depois de um processo de pesquisa sobre as propriedades físicas e mecânicas dos materiais, se desenvolveram seis protótipos com diferentes proporções, resultantes da fusão dos plásticos reciclados e a fibra de fique, determinando o comportamento mecânico dos compostos a partir de ensaios de resistência à tração, à compressão, à flexão, ao calor e à estabilidade. Assim, se determinou que a fibra não aumenta a resistência, mas sim a capacidade de se deformar sem incorrer no rompimento, o que the oferece amplas propriedades de ductilidade.

Palavras-chave: uso de resíduos plásticos; termoplásticos; pós-consumo; polietileno de alta densidade; cloruro de polivinilo; polietileno tereftalato; materiais de construção; moradia rural. 


\section{Conception d'éco-toitures en plastique recyclé et renforcé de fibres naturelles (Furcraea andina) pour améliorer les conditions de logement des populations rurales en Colombie}

\section{RÉSUMÉ}

Cette investigation tente de répondre à deux problématiques fondamentales: d'une part la contamination et les impacts environnementaux négatifs générés par l'accumulation massive de déchets plastiques et de matériaux de construction, et d'autre part, la mauvaise qualité et le déficit quantitatif et structurel de logements en zone rurale dû aux déplacements forcés, à la violences des phénomènes naturels et au manque d'investissement des pouvoirs publics. Cet article analyse les nécessaires transformations des matières premières pour générer de nouveaux matériaux au travers de l'utilisation de thermoplastiques de polyéthylène haute densité recyclés, de chlorure de polyvinyle et de polyéthylène téréphtalate renforcés de fibres naturelles pour la fabrication de matériaux de construction dans le but d'améliorer les conditions structurelles de l'habitabilité des logements ruraux. Nous avons ainsi mis en place une méthodologie scientifique et expérimentale d'approche mixte dans laquelle six prototypes de dimensions différentes ont été fabriqués, tous issus de la fusion de plastiques recyclés avec des fibres naturelles (Furcraea andina) pour analyser les comportements mécaniques des composés à partir d'essais de résistance, de traction, de compression, de flexion, de chaleur et de stabilité. Nous avons pu déterminer que la fibre n'améliore pas la résistance en soi du nouveau matériau, mais lui permet se déformer sans subir de ruptura, lui conférant ainsi de puissantes propriétés de ductilité.

Mots clés: utilisation des déchets plastiques; thermoplastiques; postconsommation; polyéthylène de haute densité; chlorure de polyvinyle; polyéthylène téréphtalate; matériaux de construction; logement rural. 


\section{Introducción}

El calentamiento global representa uno de los problemas medioambientales de mayor preocupación en la actualidad, ya que altera de manera drástica el ciclo normal de los ecosistemas. Existen dos variables fundamentales que tienen gran incidencia en la manifestación de este fenómeno. La primera es la producción y acumulación masiva de plásticos en los entornos naturales. Debido a sus características físicas y químicas, los plásticos poseen un alto índice de duración, de manera que, a priori, pueden perdurar intactos por cientos de años como basura; además, durante su degradación producen la liberación de metano y etileno, gases fundamentales en la generación del efecto invernadero.

De acuerdo con una encuesta de ING, realizada a 13137 personas de todo el mundo, los residuos plásticos se consideran el problema clave para el medio ambiente, seguido por el cambio climático, la contaminación atmosférica, el agotamiento de los recursos y la pérdida de biodiversidad (véase la Figura 1) (Geijer, 2019).

Figura 1. Porcentaje de encuestados sobre la pregunta: «¿Cuál crees que es el problema más preocupante para el medio ambiente?».

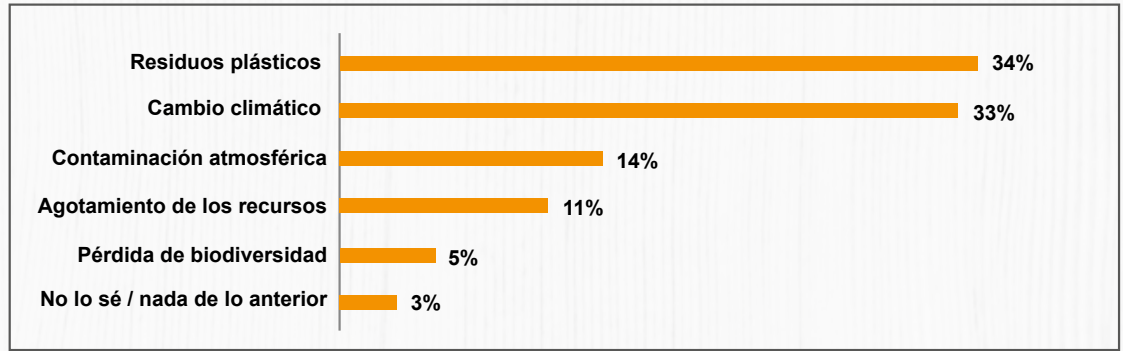

Fuente. Geijer, 2019.

Lo anterior se debe a la evolución en los procesos de industrialización, lo que ha incrementado la oferta y demanda de bienes de consumo y, consigo, la generación de residuos contaminantes y basuras, conformados, en gran parte, por desechos plásticos - suponen alrededor del $40 \%$ del neto de las 
basuras acumuladas-, de los cuales aproximadamente el $9 \%$ se recicla, el $12 \%$ se incinera y el $79 \%$ se acumula en vertederos o en entornos naturales (Geyer, Jambeck y Law, 2017).

Como segunda variable se encuentran los impactos ambientales causados por los materiales utilizados en la construcción, tales como el cemento, el acero y el concreto, ya que su producción implica el uso representativo de energía, diversos tipos de maquinarias y la explotación de recursos naturales, así como los desechos que generan. Estadísticas demuestran que el sector de la construcción es responsable de «un 12-16\% del consumo de agua; un $25 \%$ de la madera cosechada; un 30-40 \% del consumo energético; un $40 \%$ de los materiales vírgenes extraídos y un 20-30\% de las emisiones de gases de efecto invernadero» (Macozoma, 2002).

Por otra parte, el déficit de viviendas y los problemas en estas, especialmente en las poblaciones de los sectores rurales de Colombia víctimas de las crisis de desplazamiento, violencia, fenómenos naturales y la falta de atención e inversión por parte de las entidades gubernamentales, infieren de manera significativa en el desarrollo del país. Este es el caso de las zonas rurales del municipio de Tocaima, de acuerdo con el Censo de Población y Vivienda 2005: «De 1159 familias con cálculo de logros, en 1015 se presentan problemas de materiales adecuados de la vivienda, 553 tienen problemas de hacinamiento, 289 tienen pisos de tierra y en 848 hay problemas de iluminación, ventilación natural y privacidad» (Concejo Municipal de Tocaima Cundinamarca, 2012, p. 36).

A causa de los aspectos mencionados, los ingenieros se ven en la necesidad y la obligación de promover actitudes y soluciones estratégicas que forjen relaciones responsables con el medio ambiente y las poblaciones más vulnerables, por medio del diseño de proyectos de desarrollo alternativos y sostenibles que integren un equilibrio entre la economía, la sociedad, la ecología y el uso moderado y equitativo de los recursos disponibles.

En la ingeniería civil los materiales constituyen una de las áreas más importantes en el desarrollo de infraestructuras, por tanto, es de vital importancia ahondar en la tecnología de las materias primas empleadas en la industria de la construcción, optimizando los residuos reciclables y reutilizables para la generación de nuevos materiales que contribuyan de forma positiva a los impactos ambientales y optimicen los recursos económicos. 
Como propuesta para asumir estos desafíos surge una alternativa que busca sustituir las cubiertas tradicionales fabricadas en fibrocemento, arcilla o metal, entre otros, por las denominadas «ecocubiertas», diseñadas a partir del reciclaje de termoplásticos posconsumo, fabricados en polietileno de alta densidad (PEAD), cloruro de polivinilo (PVC) y polietileno tereftalato (PET) reforzados con fibra de fique - Furcraea andina-, lo cual permita la recolección y el aprovechamiento de las aguas lluvias y, con ello, mejorar las condiciones de habitabilidad de una vivienda sustentable en el sector rural de Colombia.

Para esto se tomaron como referencia diversos antecedentes nacionales e internacionales en la implementación de plásticos reciclados en el desarrollo de cubiertas, entre los cuales se resaltan las tejas desarrolladas en Brasil a partir de empaques de tetrabrik, compuestos por aluminio, cartón y plástico (Fiorelli, Vaz, Morceli y Dias, 2009); los techos patentados en Europa producidos con componentes de polietileno, polipropileno, áridos y caucho (Boor, 2009); la mezcla de partículas de caucho derivado de neumáticos desechados y polietileno reciclado para la construcción de techados en España (Navarro, Partal, Martínez-Boza y Gallegos, 2010); y el aprovechamiento de polipropileno y polietileno de alta densidad reciclados, reforzados con fibra natural-vegetal en Pasto, Colombia (Córdoba, Mera, Martínez y Rodríguez, 2010). Estos sirvieron como base para definir, analizary comparar los métodos experimentales y los comportamientos manifestados en la implementación del polietileno y las fibras naturales en la construcción de cubiertas, así como sus características mecánicas y físicas.

En la primera fase de la investigación se realizó la documentación de toda la información referente al PEAD, el PVC y el PET, su comportamiento frente al calor, su funcionalidad y resistencia, así como las características físicomecánicas de la fibra de fique. En la segunda fase, por medio del reciclaje de los plásticos posconsumo - previamente identificados- se efectuó una fusión con la fibra de fique, determinando el comportamiento mecánico de los compuestos a partir de ensayos de resistencia a la tracción, la compresión, la flexión, la estabilidad y la resistencia al calor. 
A partir de los datos obtenidos se desarrolló un análisis de la capacidad estructural que posee el material para una cubierta, cumpliendo con la carga portante y el funcionamiento requerido. Con esto se busca estar en capacidad de implementar estas innovaciones tecnológicas y reemplazar las materias primas no renovables a fin de favorecer a las comunidades más vulnerables de las zonas rurales del país, en busca de generar impactos sociales, ambientales y económicos axiomáticos en el desarrollo de la sociedad.

\section{Metodología}

A partir de la problemática expuesta y los objetivos planteados se emplea una metodología de carácter documental y científico, según el proceso de registro y documentación de la información, aplicando un diseño metodológico experimental por medio de la caracterización y la experimentación para el desarrollo y la construcción del material propuesto. Lo anterior bajo un enfoque mixto, de acuerdo con el análisis estructural necesario para determinar el comportamiento mecánico del producto final. En este sentido, la investigación se desarrolló en tres fases: recolección y presentación de la información, proceso constructivo y experimental para la elaboración de los prototipos, y el análisis e interpretación estructural de los resultados obtenidos en el laboratorio.

En la elaboración de los prototipos de las cubiertas se emplearon tres plásticos fundamentales en el marco de los residuos: el PEAD, obtenido de botellas de detergentes y productos de limpieza; el PVC, proveniente de botellas de aceites y residuos de construcción; y el PET, obtenido de botellas de gaseosas, jugos, aguas y similares.

El componente utilizado como refuerzo fue fibra de fique, procedente de las plantas de género Furcraea andina. Inicialmente, las fibras se sometieron a un tratamiento de alcalinización en disolución acuosa de hidróxido de sodio $(\mathrm{NaOH})$, en relación del $2 \% \mathrm{p} / \mathrm{v}$ a $25^{\circ} \mathrm{C}$ por una hora; luego se lavaron con agua destilada con la finalidad de remover el sobrante de $\mathrm{NaOH}$. De forma consecutiva, el material se secó en dos fases: primero, a temperatura ambiente durante 12 horas, y subsiguientemente en horno a $60{ }^{\circ} \mathrm{C}$ por 24 horas. Este 
proceso tiene como objetivo aumentar la resistencia máxima y el módulo de elasticidad de la fibra. Posteriormente, las fibras fueron trituradas por un molino de cuchillas y se tamizaron, con lo cual se obtuvo tamaños de 1 a $3 \mathrm{~mm}$ $y$, finalmente, el material de refuerzo a fin de aplicarlo en la elaboración de los prototipos.

Para el aprovechamiento de los plásticos de PEAD, PVC y PET se empleó la técnica de reciclaje mecánico, la cual consiste en seis fases: recolección, clasificación y selección, molienda, lavado, enjuague, y filtrado y secado. En este orden, los materiales previamente seleccionados y acondicionados fueron triturados hasta obtener fragmentos de tamaños variables entre 2 a $6 \mathrm{~mm}$ y, de forma consecutiva, se lavaron con cloro y una solución detergente con efectos desengrasantes con el propósito de eliminar los residuos contaminantes. Posteriormente, el material fue secado, de modo que se eliminó su humedad por completo.

En el diseño de las mezclas se realizaron pruebas de proporciones aleatorias experimentales con mezclas de PEAD, PVC y PET en porcentajes de $33,3-33,3-33,3,25-50-25,25-60-15$, respectivamente, sin fibra y con $5 \%$ de fibra en relación con el peso empleado en cada material, para un total de seis especímenes (véase la Tabla 1). En este sentido, se escogieron las proporciones de acuerdo con las propiedades de cada material, por lo que el PVC se mantiene en las seis muestras como el de mayor porcentaje, ya que este manifiesta propiedades mecánicas superiores en cuánto a resistencia, seguido por el PEAD y, por último, el PET. En cuanto a la fibra de fique, se realizan tres muestras sin fibra y tres con el $5 \%$ con el fin de determinar la interacción de la fibra con los polímeros y cómo se comporta el material cuando no presenta fibra; además, de esta forma establecer su efectividad como refuerzo polimérico.

Tan pronto se realizó la proporción indicada de cada material se dejó a un lado la fibra y se mezclaron, de manera uniforme, en un recipiente metálico, los tres materiales plásticos particulados según el porcentaje de cada espécimen; una vez lista la composición, se llevaron las muestras a un horno a temperaturas de entre 250 y $270{ }^{\circ} \mathrm{C}$ hasta obtener una mezcla líquida completamente maleable y homogénea. De inmediato, se retiró la muestra del horno, se añadió el porcentaje de fibra correspondiente y se mezcló hasta que 
quedó uniforme, para, posteriormente, transportarla y disponerla en moldes cerámicos en donde adquirieron la forma de ondulación de la teja, en cuatro capas, cada una de $1 \mathrm{~mm}$. Rápidamente, los especímenes fueron secados por medio de secadores con aire a temperatura ambiente durante unos 30 a $40 \mathrm{~min}$, luego se desmoldaron y se dispusieron en un bastidor de madera evitando que se generarán deformaciones o fracturas y se dejaron reposar a temperatura ambiente durante $48 \mathrm{~h}$, hasta que se solidificarán y enfriarán por completo. Los especímenes finalmente secos y rígidos en forma de rectángulo ondulado poseen una masa que varía de $1,14 \mathrm{~kg}$ a $1,18 \mathrm{~kg}$ (véase la Tabla 1) con las siguientes características geométricas: ancho de $400 \mathrm{~mm}$; largo de 500 $\mathrm{mm}$; espesor de $4 \mathrm{~mm}$.

Tabla 1. Proporciones de materiales para los diferentes tipos de mezcla.

\begin{tabular}{|c|c|c|c|c|c|}
\hline Espécimen & PEAD (\%) & PVC (\%) & PET (\%) & $\begin{array}{c}\text { Fibra de } \\
\text { fique (\%) }\end{array}$ & Masa (kg) \\
\hline Espécimen N ${ }^{\circ} 1$ & 33,3 & 33,3 & 33,3 & 0 & 1,156 \\
\hline Espécimen N $\mathrm{N}^{\circ}$ & 33,3 & 33,3 & 33,3 & 5 & 1,164 \\
\hline Espécimen $\mathrm{N}^{\circ} 3$ & 25 & 50 & 25 & 0 & 1,14 \\
\hline Espécimen $\mathrm{N}^{\circ} 4$ & 25 & 50 & 25 & 5 & 1,152 \\
\hline Espécimen $\mathrm{N}^{\circ} 5$ & 25 & 60 & 15 & 0 & 1,169 \\
\hline Espécimen $\mathrm{N}^{\circ} 6$ & 25 & 60 & 15 & 5 & 1,18 \\
\hline
\end{tabular}

Fuente. Elaboración propia.

Al finalizar el proceso de diseño de las mezclas se procedió a realizar las respectivas pruebas mecánicas tomando como referencia la NTC 3201 «Métodos de ensayo normalizados para determinar las propiedades de flexión de plásticos reforzados y no reforzados y de materiales aislantes eléctricos». Estos consisten en ensayos de resistencia a la compresión, resistencia a la flexión, resistencia a la tensión y resistencia al impacto para cada uno de los especímenes, llevados a cabo en una máquina universal en la Universidad Piloto de Colombia, Seccional Alto Magdalena, en el laboratorio de resistencia de materiales. De igual forma, se desarrollaron pruebas de absorción al agua. Todo esto con la finalidad de verificar que el material sea resistente a grandes cargas y agentes climáticos extremos tales como lluvias, fuertes vientos y elevadas temperaturas. 


\section{Discusión y análisis de resultados}

Los especímenes desarrollados poseen un peso menor comparado con las tejas tradicionales, con una masa que va desde $1,14 \mathrm{~kg}$ a $1,18 \mathrm{~kg}$ para cada muestra, con un espesor de $4 \mathrm{~mm}$, lo cual brinda una característica fundamental a la hora de implementarlo en una vivienda, puesto que van a tener menor incidencia de carga en las estructuras y cimentaciones que las soporta, con lo cual, además, se ahorra en costos y materiales.

A fin de determinar si los prototipos son aptos para ser empleados como la estructura de cubierta de una vivienda se desarrolló el análisis estructural de los ensayos de resistencia mecánica por medio del Diagrama esfuerzodeformación unitaria, el cual proporciona el esfuerzo necesario para que el material fluya plásticamente hacia cualquier deformación dada e identificar cómo es su comportamiento mecánico.

En la figura 2 se muestra el comportamiento a la flexión para cada espécimen según sus respectivas proporciones. Allí se puede observar cómo las muestras que no presentan fibra disminuyen de manera significativa su resistencia en comparación con aquellas que presentan el $5 \%$, pero se manifiesta una gran deformación en el material, con decremento en la carga aplicada. Así mismo, se determinó que el material en su punto máximo de resistencia, antes de continuar con su deformación, es capaz de soportar cargas a la flexión de hasta $3000 \mathrm{~N}$ con un aumento representativo en la ductilidad del material sin que se genere una falla súbita. 
Figura 2. Diagrama de esfuerzo a la flexión de los especímenes.

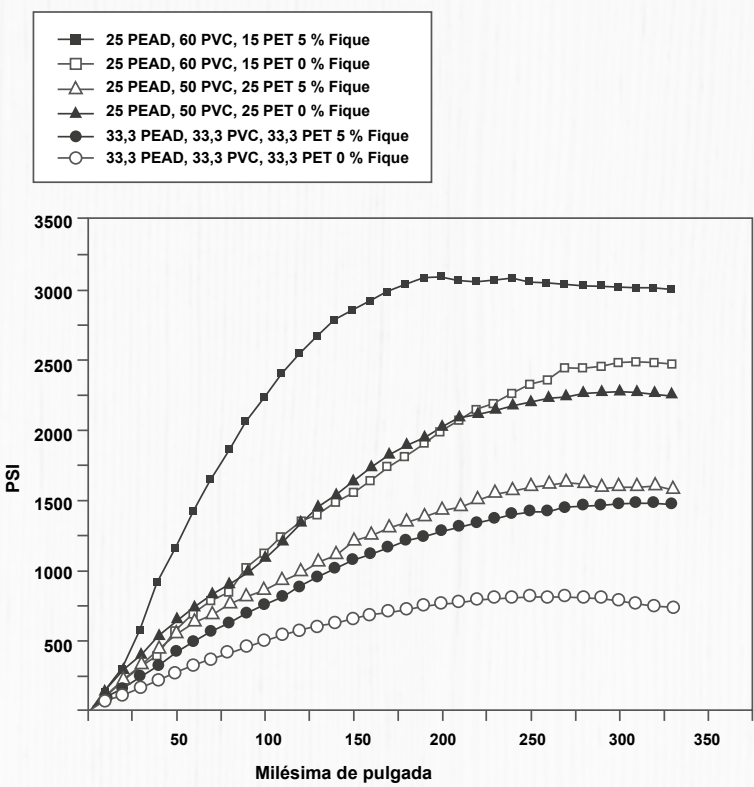

Fuente. Elaboración propia.

En cuanto al comportamiento a la compresión, en la figura 3 se puede observar que las proporciones con contenido del $5 \%$ de fibra manifiestan una disminución en la resistencia y en el módulo, pero con un incremento en su ductilidad. La fibra le proporciona a la mezcla una tendencia a la rotura menor y, en el momento en que se genera la deformación, a medida que se incrementa la carga, se revela una mayor estabilidad por parte del elemento. Este aumento de ductilidad en los comportamientos de flexión y compresión se puede deber a que las cadenas moleculares de los plásticos, cuando se encuentran a bajos niveles de deformación, tienen el tiempo suficiente para alinearse entre ellas bajo la influencia de la carga aplicada, lo que le brinda esa estabilidad elástica al material. 
Figura 3. Diagrama de esfuerzo a la compresión de los especímenes.

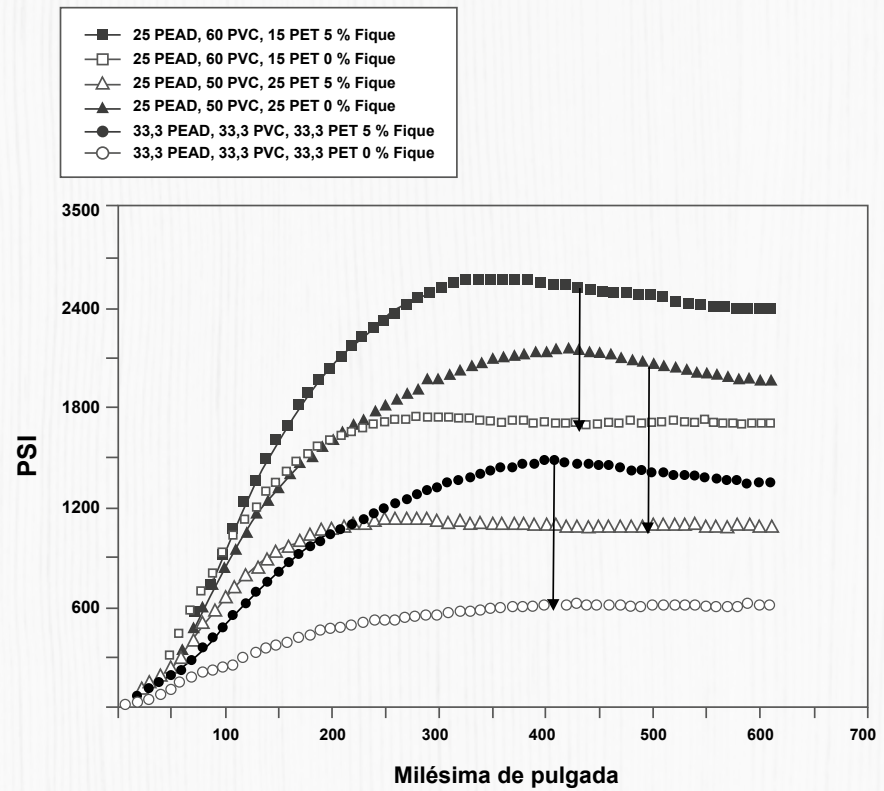

Fuente. Elaboración propia.

En cuanto al comportamiento a la tensión, tal como se puede identificar en la figura 4, la resistencia disminuye cuando se le aplica fibra al material. Ahora bien, cuando posee el porcentaje de fibra su deformación aumenta, sobre todo en la proporción 25 \% PEAD, 50 \% PVC, 25 \% PET y 5 \% fibra. Esto puede originarse debido a una falta de uniformidad en la mezcla de los componentes de la fibra con los polímeros, lo que podría generar la formación de vacíos en la mezcla y, por tanto, una disminución de la resistencia al estiramiento del material. Lo anterior sugiere que se deben realizar modificaciones en el procedimiento constructivo de unión entre los polímeros y la fibra. 
Figura 4. Diagrama de esfuerzo a la tensión-tracción de los especímenes.

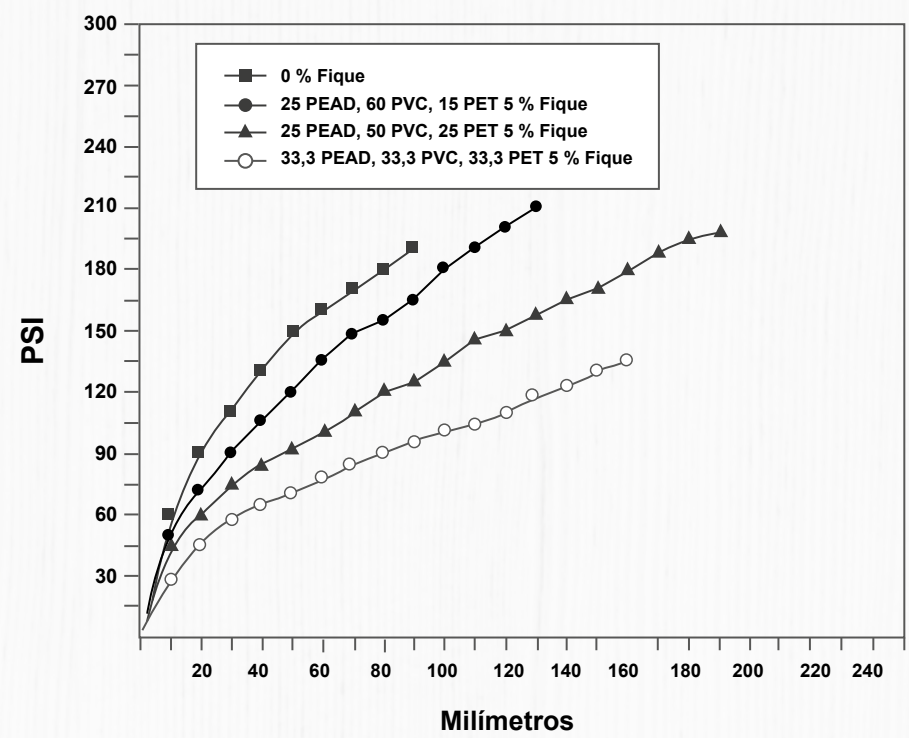

Fuente. Elaboración propia.

A partir de estos resultados se determinó que la fibra no aumenta la resistencia, pero sí la capacidad de deformarse sin incurrir en la rotura. En este sentido, podrían experimentarse modificaciones en las muestras, reduciendo el porcentaje de fibra de fique a un $4 \%$ y $3 \%$. Además, podría probarse un espécimen con un material aglutinante que aporte un porcentaje de resistencia. Así mismo, pueden emplearse otros materiales reciclables, como, por ejemplo, el aluminio o el caucho -empleados en los antecedentes referenciados-, en conjunto con el PEAD, el PVC y el PET, así como la fibra de fique, e identificar cómo inciden en el comportamiento estructural de los compuestos desarrollados, en busca de mejorar las características mecánicas de estos.

Para la resistencia al impacto, el prototipo $\mathrm{N}^{\circ} 6-25 \%$ PEAD, $60 \%$ PVC, $15 \%$ PET y $5 \%$ de fibra-manifestó un comportamiento favorable al choque instantáneo y a variaciones de una carga externa aplicada por largos periodos de tiempo, puesto que no mostraron daños superficiales tales como astillados, microfisuración superficial, cráteres, fracturas ni grietas, lo cual las hace aptas para soportar cargas externas y climáticas. 
Por lo anterior, se determinó que el espécimen $\mathrm{N}^{\circ} 6$, con proporciones $25 \%$ PEAD, $60 \%$ PVC, $15 \%$ PET y $5 \%$ de fibra, manifestó un comportamiento a flexión, compresión e impacto superior a las otras mezclas, con una resistencia a cargas de hasta $3000 \mathrm{~N}$ y un aumento en la ductilidad del material. Por su parte, el espécimen $\mathrm{N}^{\circ} 1$, de proporciones $33,3 \%$ PEAD, $33,3 \%$ PVC, 33,3 \% PET y $0 \%$ de fibra, fue el de menor resistencia.

Con respecto a las pruebas dirigidas a establecer la absorción al agua de los especímenes, estos fueron dispuestos en un recipiente con agua durante 24 horas. Manifestaron un comportamiento adecuado debido a que absorbieron una cantidad de agua despreciable, con un porcentaje de hasta $0,24 \%$, esto gracias a las propiedades que aporta cada uno de los materiales que, al estar en su estado seco no permiten el paso ni la absorción de agua sin alterar su masa inicial.

Tabla 2. Proporciones de materiales para los diferentes tipos de mezcla.

\begin{tabular}{|c|c|c|c|c|c|}
\hline Espécimen & $\begin{array}{c}\text { PEAD } \\
(\%)\end{array}$ & $\begin{array}{c}\text { PVC } \\
(\%)\end{array}$ & $\begin{array}{c}\text { PET } \\
(\%)\end{array}$ & $\begin{array}{c}\text { Fibra de } \\
\text { fique (\%) }\end{array}$ & $\begin{array}{c}\text { Absorción } \\
\text { al agua (\%) }\end{array}$ \\
\hline Espécimen $\mathrm{N}^{\circ} 1$ & 33, & 33,3 & 33,3 & 0 & 0,234 \\
\hline Espécimen $\mathrm{N}^{\circ} 2$ & 33,3 & 33,3 & 33,3 & 5 & 0,24 \\
\hline Espécimen $\mathrm{N}^{\circ} 3$ & 25 & 50 & 25 & 0 & 0,231 \\
\hline Espécimen $\mathrm{N}^{\circ} 4$ & 25 & 50 & 25 & 5 & 0,238 \\
\hline Espécimen $\mathrm{N}^{\circ} 5$ & 25 & 60 & 15 & 0 & 0,222 \\
\hline Espécimen $\mathrm{N}^{\circ} 6$ & 25 & 60 & 15 & 5 & 0,229 \\
\hline
\end{tabular}

Fuente. Elaboración propia.

De igual forma, el material desarrollado tiene una gran eficiencia como aislante térmico, una alta resistencia al fuego y a los productos químicos, propiedades aportadas por las características físicas de los materiales reciclados. Además, presenta una gran impermeabilidad al agua y al aire. 


\section{Conclusiones}

De acuerdo con los resultados obtenidos se puede establecer que el prototipo diseñado es apto para su implementación como estructura de cubierta en una vivienda sustentable del sector rural, ya que, estructuralmente, manifestó comportamientos favorables en cuanto a resistencia y durabilidad, así como bajo peso y ductilidad, lo que favorece su funcionalidad. Así mismo, se identificó que presenta propiedades aislantes al calor, lo que favorece la habitabilidad de las viviendas en zonas de altas temperaturas, así como resistencia al fuego, a productos químicos e impermeabilidad de agentes climáticos, lo que le permite comportarse como una barrera de seguridad ante agentes climáticos tales como lluvias, fuertes vientos y altas temperaturas.

Con respecto a los ensayos, los conocimientos y las experiencias obtenidas durante la investigación, se concluyó que el material con proporción $25 \%$ de PEAD, $60 \%$ de PVC, $15 \%$ de PET y $5 \%$ de fibra de fique fue el que presentó mejores resultados mecánicos, obteniendo resistencias mayores a las demás mezclas, puesto que se empleó una mayor cantidad de PVC y PET como el menor porcentaje debido a las propiedades físico-mecánicas determinadas previamente. En cuanto a los comportamientos a la flexión, compresión y tensión, se determinó que la fibra no aumenta su resistencia, pero sí su capacidad de deformarse sin incurrir en la rotura, lo cual puede aprovecharse para elementos que soliciten ser más dúctiles y livianos.

Como conclusión final, se puede establecer que los especímenes realizados poseen varias ventajas que orientan a un desarrollo sostenible en tres factores fundamentales: el social, el ambiental y el económico. El social, ya que el desarrollo de estas cubiertas tiene como finalidad básica mejorar las condiciones de habitabilidad de las viviendas, principalmente, de aquellas en donde habitan las comunidades más vulnerables que no tienen acceso a una estructura digna y adecuada. El ambiental, puesto que las cubiertas son ecológicas, contribuyen a la gestión de residuos, al aprovechamiento de los recursos y las materias primas disponibles, generando con ello distintas alternativas en el sector de la elaboración de materiales constructivos. Por último, el económico, debido a que la producción de estas cubiertas contribuye 
a la generación de empleo, apoya la labor de sectores económicos olvidados como lo son, por ejemplo, los recicladores, en busca de una alianza entre los trabajadores de este medio para que sean ellos los principales proveedores de la materia prima necesaria.

Por otra parte, para la academia, así como para el sector de la construcción y los campos ambientales afines, esta investigación puede ser un punto de partida en el propósito de crear una opción viable en la gestión y reutilización de los residuos plásticos, añadiendo a esto un aporte significativo a las poblaciones rurales de Colombia.

\section{Reconocimientos y agradecimientos}

Este artículo está dedicado a todos los jóvenes de escasos recursos que no cuentan con los medios suficientes para tener una educación superior óptima y viven en condiciones de vida luctuosas, a fin de que sigan adelante a pesar de las adversidades y luchen por sus sueños sin importar las barreras que se presenten.

Agradezco profundamente a mi madre, mi hermana, mis abuelos, mi padre y mi tía, quienes me han apoyado incondicionalmente durante todo mi proceso de aprendizaje, apoyándome y ayudándome en todo lo necesario.

De igual forma, agradezco a la Universidad Piloto de Colombia, SAM, al semillero de investigación Sentram y a mi docente Ancizar Barragán, quienes me han guiado y prestado las herramientas necesarias para estar en capacidad de desarrollar adecuadamente este trabajo. Agradezco a Dios y su grandeza, sabiduría y bondad. 


\section{Referencias}

Boor, B. J. (2009). Wege Road, Hortonville Patente $n^{\circ} 20090308009$.

Concejo Municipal de Tocaima Cundinamarca. (2012). Plan de desarrollo 2012-2015, «Inclusión para la prosperidad de Todos». Tocaima, Colombia: Municipio de Tocaima.

Cordoba, C.; Mera, J.; Martínez, D.; Rodríguez, J. (2010). Aprovechamiento de polipropileno y polietileno de alta densidad, reforzados con fibra vegetal, tétera (Stromanthe Stromathoides). Revista Iberoamericana de Polímeros, 11(7), 417-427. Recuperado de https://bit.ly/2EAGuYI

Fiorelli, J.; Vaz, R.; Morceli, J.; \& Dias, A. (2009). Avaliação da eficiência térmica de telha reciclada à base de embalagens longa vida. Revista Brasileira de Engenharia Agrícola e Ambiental, 13(2), 204-209. DOI: https://doi. org/10.1590/s1415-43662009000200015

Geijer, T. (3 de diciembre de 2019). Plastic packaging in the food sector. Six ways to tackle the plastic puzzle. ING THINK Economic and Financial Analysis. Recuperado de https://bit.ly/3ldzfXM

Geyer, R.; Jambeck, J. R.; Law, K. L. (2017). Production, use, and fate of all plastics ever made. Science Advances, 3(7). 1-5. DOI: https://doi. org/10.1126/sciadv.1700782

Macozoma, D. S. (2002). Construction site waste management and minimization. Reporte preparado para el International Council for Research and Innovation in Building and Construction (CIB). Recuperado de https://bit. ly/3hpIVfB

Navarro, F. J.; Partal, P.; Martínez-Boza, F. J.; Gallegos, C. (2010). Novel recycled polyethylene/ground tire rubber/bitumen blends for use in roofing applications: Thermo-mechanical properties. Polymer Testing, 29(5), 588595. DOI: https://doi.org/10.1016/j.polymertesting.2010.03.010 